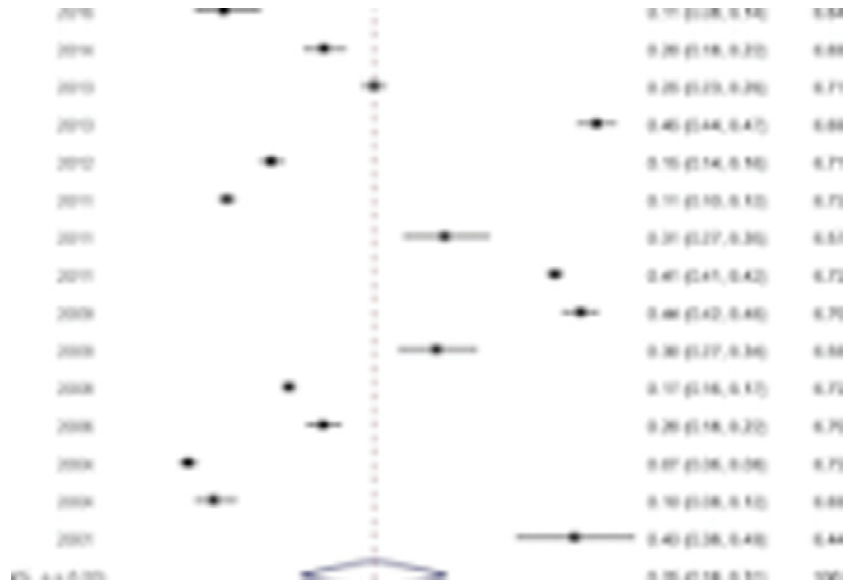

review, in particular, related to the sample size used in each study. Two facts must be accounted for, first the statistical difficulties associated to the estimation of small prevalence and the consequent heterogeneity of the estimates, and, second, the limited number of studies included in this meta-analysis. Nonetheless, there is evidence about big heterogeneity what can correspond to non-observed variables, in particular, life-styles, and environmental or genetic traits.

Disclosure of Interest: None declared

DOI: 10.1136/annrheumdis-2017-eular.3919

\section{FRI0710 MONOSODIUM URATE CRYSTAL DEPOSITION ASSOCIATED WITH THE CHANGE OF RADIOGRAPHIC GRADE AT THE SACROILIAC JOINT IN AXIAL SPA: A DUAL-ENERGY CT STUDY}

J. Zhu $^{1}$, A. Aiwu Li ${ }^{2}$, E. Jia ${ }^{2}$, Y. Zhou ${ }^{3}$, J. Xu$^{2}$, S. Chen ${ }^{2}$, Y. Huang ${ }^{2}$, X. Xiao ${ }^{4}$, J. Li ${ }^{2} .{ }^{1}$ Department of Rheumatology, Nanfang Hospital, Southern Medical University; ${ }^{2}$ Department of Internal Medicine of Traditional Chinese Medicine, College of Traditional Chinese Medicine, Southern Medical University; ${ }^{3}$ Department of Obstetrics, Guangdong Women and Children Hospital; ${ }^{4}$ Department of Radiology, Nanfang Hospital, Southern Medical University, Guangzhou, China

Background: Previous studies have revealed that ankylosing spondylitis (AS), as the progenitor of axial spondyloarthritis (AxSpA), has been characterized by the insidiously progressive nature of sacroiliitis and spondylitis. Dual-energy computed tomography (DECT) has recently been used to analyse the deposition of monosodium urate (MSU) crystals with higher sensitivity and specificity. However, it remains unclear whether the existence of the MSU crystal deposits detected by DECT at the sacroiliac joint in patients with AxSpA also contributed to the existing structural damage.

Objectives: We performed this study to show the DECT MSU crystal deposits in AxSpA patients without coexisting gout and to ascertain whether the MSU crystal deposits at the sacroiliac joint in those patients increased the risk of the structural joint damage.

Methods: One hundred and eighty-six AxSpA patients without coexisting gout were recruited. The plain radiographs of the sacroiliac joint were obtained, along with the DECT scans at the pelvis and the clinical variables. All statistics based on the left or right sacroiliac joint damage grading (0-4) were calculated independently. Bivariate analysis and ordinal logistic regression was performed between the clinical features and radiographic grades at the sacroiliac joint.

Results: At painful joints or skeleton regions, large quantities of MSU crystal deposits were found in 186 patients with $\mathrm{AxSpA}$, as depicted in green with DECT. The average MSU crystal volume at the left sacroiliac joint, the right sacroiliac joint, and the pelvis were $0.902 \pm 1.345,1.074 \pm 1.878$, and $5.272 \pm 9.044$ $\mathrm{cm}^{3}$, values which were correlated with serum uric acid concentrations $(r=0.727$, $0.740,0.896 ; p<0.001)$. At the left and right sacroiliac joint, the presence of MSU crystal deposits $(=11.451,43.684 ; p<0.01)$ and the volumes of MSU crystals ( $Z=9.198, Z=34.607 ; p<0.05)$ were statistically different among groups divided by the ASDAS scores. In bivariate analysis, wide clinical variables were associated with the changes in sacroiliac joint damage. When others factors were adjusted in the ordinal logistic models, the AxSpA duration, total back pain, BASFI score, and volume of MSU crystallization were the risk factors for the radiographic grade at the left sacroiliac joint $(\mathrm{AOR}=1.187,1.428,3.837,2.018 ; p<0.05)$. The same risk factors were obtained for the right sacroiliac joint, except for total back pain. Additionally, the simplified models excluded the repeated variables; the AxSpA duration, BASFI score, and the volume of MSU crystallization at both sides of sacroiliac joint served as risk factors for the radiographic grade (left-AOR=1.180, $3.800,1.920$; right-AOR=1.190, 3.034, 1.418; $p<0.01$ ).

Conclusions: Large quantities of MSU crystal deposits detected by DECT were found in AxSpA patients without coexisting gout. In addition to AxSpA duration and BASFI score, the MSU crystal deposits at the sacroiliac joint in those patients independently increased the risk of structural joint damage.

Disclosure of Interest: None declared
DOI: 10.1136/annrheumdis-2017-eular.1417

\section{FRI0711 DISCORDANCE OF THE FRAMINGHAM CARDIOVASCULAR RISK SCORE AND THE 2013 AMERICAN COLLEGE OF CARDIOLOGY/AMERICAN HEART ASSOCIATION RISK SCORE IN SYSTEMIC LUPUS ERYTHEMATOSUS AND RHEUMATOID ARTHRITIS}

K. Jafri ${ }^{1}$, A. Ogdie ${ }^{2}$, P. Katz ${ }^{1}$, J. Yazdany ${ }^{1}$, G. Schmajuk ${ }^{1,3} \cdot{ }^{1}$ Rheumatology, University of California, San Francisco, San Francisco; ${ }^{2}$ Rheumatology, University of Pennsylvania, Philadelphia; ${ }^{3}$ Rheumatology, Veterans Affairs Medical Center, San Francisco, United States

Background: Systemic lupus erythematosus (SLE) and rheumatoid arthritis (RA) are associated with an increased risk of cardiovascular (CV) disease, and multipliers to traditional 10-year CV risk scores, such as a EULAR-recommended 1.5 multiplier in RA, have been proposed to capture this increased CV risk. The discordance between CV risk assessment by the Framingham risk score, a modified Framingham risk score (with a 1.5 multiplier), and the more recent 2013 American College of Cardiology/American Heart Association (ACC/AHA) risk score has not been well-studied in patients with rheumatic diseases.

Objectives: To determine the proportion of discordant 10-year Framingham risk scores and 2013 ACC/AHA risk scores in subjects with SLE and RA, both with and without a 1.5 multiplier to the Framingham risk score, and to assess demographic, $\mathrm{CV}$, and rheumatologic clinical characteristics associated with discordant risk scores.

Methods: A cross-sectional study was conducted using SLE and RA subjects drawn from the University of California, San Francisco, Arthritis, Body Composition, and Disability project. 10-year Framingham risk scores, modified Framingham risk scores (with a 1.5 multiplier), and 2013 ACC/AHA risk scores were calculated. As per Adult Treatment Panel-III (ATP-III) recommendations, a subject with a Framingham risk score (or modified Framingham risk score) $\geq 10 \%$ was defined as high-risk by that score, whereas a subject with a Framingham risk score (or modified Framingham risk score) $<10 \%$ was defined as low-risk. A subject with a 2013 ACC/AHA risk score $>7.5 \%$ was defined as high-risk by that score, whereas a subject with a 2013 ACC/AHA risk score $<7.5 \%$ was defined as low-risk. A subject with a discordant risk score was defined as one who had a Framingham risk score (or modified Framingham risk score) that characterized him/her as low-risk and a 2013 ACC/AHA risk score that characterized him/her as high risk. Associations of demographic, $\mathrm{CV}$, and rheumatologic characteristics with discordant risk scores were analyzed using chi-squared tests for categorical variables and using independent $t$-tests for continuous variables.

Results: $11(7.0 \%)$ of the 157 SLE subjects and $11(11.5 \%)$ of the 96 RA subjects had discordant $\mathrm{CV}$ risk scores with low Framingham risk scores but high ACC/AHA risk scores. When the 1.5 multiplier was applied to the Framingham risk score, the number of subjects with discordant risk scores did not significantly change. Rheumatologic disease duration, CRP levels, African-American race, diabetes, current use of anti-hypertensive medication, higher age, and higher systolic blood pressure were all significantly associated with discordant risk scores.

Conclusions: Approximately $10 \%$ of SLE and RA subjects had discordant 10-year $\mathrm{CV}$ risk scores with low Framingham risk scores but high ACC/AHA risk scores, even when a 1.5 multiplier was applied to the Framingham risk score. Prospective studies are needed to address the ability of different CV risk assessment tools, such as the 2013 ACC/AHA risk score, Framingham risk score, and modified risk scores, to predict $\mathrm{CV}$ events in rheumatologic patients, especially those with risk factors associated with discordant risk scores.

Disclosure of Interest: None declared

DOI: 10.1136/annrheumdis-2017-eular.1171

\section{FRI0712 DRIVERS OF UNREFRESHING SLEEP IN PEOPLE WITH MUSCULOSKELETAL PAIN}

K.L. Druce, J. McBeth. Arthritis Research UK Centre for Epidemiology, The University of Manchester, Manchester, United Kingdom

Background: Waking feeling unrefreshed is associated with poor health outcomes including an increased risk of cardiovascular death. Pain is a robust predictor of waking feeling unrefreshed. Pain is a complex disorder and it is not clear whether the pain itself, or associated somatic symptoms, mental health conditions and lifestyle factors, predicts waking unrefreshed.

Objectives: To investigate whether reporting pain was an independent predictor of waking unrefreshed among people with musculoskeletal pain.

Methods: Participants in a population study completed the Estimation of Sleep Problems Scale (ESPS), which indicates the number of days in the past month participants have experienced unrefreshing sleep, problems with sleep onset, maintenance and night awakenings. Pain assessments (body map and duration $>3$ months) were used to classify participants as having no pain, acute pain, chronic pain and CWP (ACR criteria: pain lasting $\geq 3$ months in the axial skeleton and contralateral body quadrants). Participants also reported demographics (date of birth, sex, English Index of Multiple Deprivation); somatic symptoms (Chalder Fatigue Scale (CFQ 11) and Inflammatory Bowel Syndrome (IBS)); mental health (Hospital Anxiety and Depression (HAD) scale); disability (Stanford Health Assessment Questionnaire (HAQ)) and lifestyle factors (average number 
of alcoholic drinks per week, smoking status and Rapid Assessment of Physical Activity (RAPA)). Univariable ordinal logistic regression tested the relationship between pain and waking unrefreshed. The model was then cumulatively adjusted for sleep, somatic symptoms, mental health, disability and lifestyle domains. All models were age, sex and deprivation adjusted. The results of a complete case analysis were comparable to those which used multiple imputation for missing data and the results of the complete case analyses are shown. Results were expressed as odds ratios (OR) with $95 \%$ confidence intervals $(\mathrm{Cl})$.

Results: Of 1913 people who had complete data, $1376(72 \%)$ woke unrefreshed on at least one day in the past month $(41 \% 1-7$ days; $31 \%>8$ days). Compared to those with no pain, people with acute pain and chronic pain were two (OR $2.0,95 \% \mathrm{Cl}(1.5-2.6)$ and $2.2(1.9-2.7)$, respectively) times more likely to wake unrefreshed; those with CWP were five (5.0 (4.0-6.3)), times more likely to wake unrefreshed. Following adjustment for all other variables, the relationship between reporting chronic pain (1.5 (1.2-1.8)) or CWP (1.9 (1.4-1.5)) and waking unrefreshed was attenuated, but remained statistically significant. The reporting of acute pain was not an independent predictor of waking unrefreshed (1.4 (0.981.9)). Problems with sleep onset ( $>8$ days vs $0: 2.9(2.0-4.1)$ ) and maintenance ( $\geq 8$ days vs $0: 5.9(4.1-8.4)$ ), night awakenings ( $\geq 8$ days vs $0: 2.5$ (1.7-3.7)), IBS (2.2 (1.2-4.0)), currently smoking (vs never: $1.5(1.02-2.1))$ and engagement in activities such as stretching and yoga (vs none: $0.8(0.6-0.97))$, physical $(1.3$ (1.2-1.3)) but not mental $(0.99(0.9-1.1))$ fatigue, and anxiety (definite vs no: 2.4 $(1.8-3.1)$ ), but not depression (definite vs no: $1.5(0.8-2.6)$ ), were associated with waking unrefreshed.

Conclusions: This study suggests that among people with chronic pain, the risk of waking feeling unrefreshed may be reduced through interventions that target factors such as smoking cessation, IBS management, physical fatigue and anxiety.

Acknowledgements: J. Anderson, M. Mulvey, A. Rashid

Disclosure of Interest: None declared

DOI: 10.1136/annrheumdis-2017-eular.1798

\section{FRI0713 LOW BONE MINERAL DENSITY IS THE MAIN CONTRIBUTOR TO FALLS-RELATED HEALTH BURDEN IN THE EUROPEAN ELDERLY}

L. Sanchez-Riera ${ }^{1}$, N. Wilson ${ }^{2}$, D. Prieto-Alhambra ${ }^{3}$, C. Cooper ${ }^{4}$, K. Dreinhöfer ${ }^{5}$, A. Woolf ${ }^{6}$, L. March ${ }^{2}$, P. Halbout ${ }^{7} .{ }^{1}$ University Hospital Bristol NHS Foundation Trust, Bristol, United Kingdom; ${ }^{2}$ Institute of Bone and Joint Research, University of Sydney, Sydney, Australia; ${ }^{3}$ Oxford NIHR Musculoskeletal Biomedical Research Unit, University of Oxford, Oxford; ${ }^{4}$ MRC Lifecourse Epidemiology Unit, University of Southampton, Southampton, United Kingdom; ${ }^{5}$ Center for Musculoskeletal Surgery, Charité Universitätsmedizin, Berlin, Germany; ${ }^{6}$ Institute of Health Research, University of Exeter Medical School, Exeter, United Kingdom; ${ }^{7}$ International Osteoporosis Foundation, Nyon, Switzerland

Background: Falls are the leading injury type in elderly populations and a major health burden and cause of death globally. Most of such burden is due to bone fractures. In the Global Burden of Diseases (GBD) Initiative, the attributable burden of falls due to low bone mineral density (BMD) was analysed through its relationship with fractures.

Objectives: To measure the percentage of disability-adjusted life years (DALYs), years lived with disability (YLDs) and deaths due to falls attributable to low BMD in European population for the year 2015.

Methods: The estimates followed the Counterfactual Risk Assessment Methodology used in the GBD study (1). Systematic review was performed seeking population-based studies with femoral neck (FNBMD) measured by Dual-X-RayAbsorptiometry in people 50 years and over. Age- and sex- specific levels of mean +/-SD FNBMD $\left(\mathrm{g} / \mathrm{cm}^{2}\right)$ were extracted from eligible studies, and this was used as the exposure variable. The age and sex-specific 99th percentile from non-Hispanic whites in the National Health and Nutrition Examination Survey (NHANES) 2009-2010 was used as theoretical minimum risk factor exposure distribution, to estimate the potential impact fraction (PIF) of FNBMD for fractures. Relative risks of FNBMD for fractures were obtained from a previous meta-analysis (2). Coded hospital data was used to calculate the fraction of falls-related deaths due to fractures. Disability levels were established by applying disability weights

Percentage of Deaths, DALYs and YLDs of Falls, Attributable to Low BMD in Europe

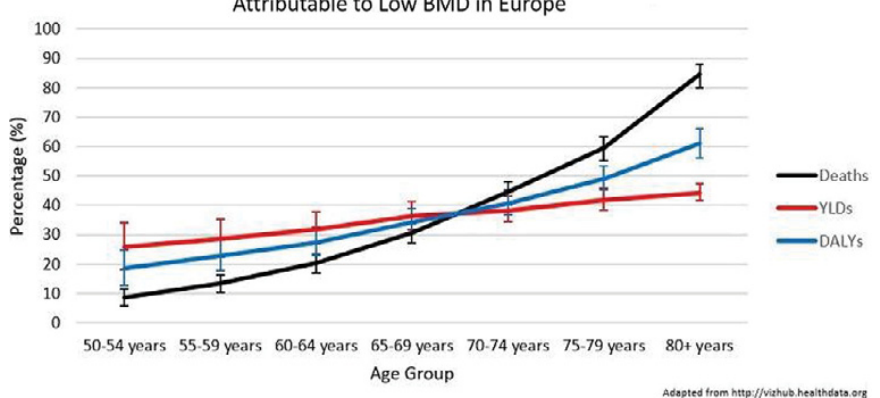

to each type of fracture. Then, PIFs were applied to obtain attributable deaths and disability due to low BMD.

Results: The percentage of falls-related preventable deaths attributable to low BMD is around $9 \%$ in the $50-54$ age group, increasing to $84 \%$ in those aged 80 years and over. Total health burden (DALYs) and disability attribution (YLDs) also increase with age, from $19 \%$ and $25 \%$ in $50-54$ years old, respectively, to $61 \%$ and $44 \%$ and population aged 80 years and above, respectively. Low BMD constitutes the most important preventable risk factors for falls-related DALYs from 50 years and above, followed by alcohol, occupational risk and smoking.

Conclusions: Low BMD is a major preventable risk factor that explains a very remarkable proportion of falls health burden in Europe, in particular in those aged 70 years and above. This is a growing concern, given the population trajectories, and requires urgent attention.

References:

[1] Forouzanfar $\mathrm{M}$ et al, Lancet 2016.

[2] Johnell O et al, JBMR 2015.

Disclosure of Interest: None declared

DOI: 10.1136/annrheumdis-2017-eular.6616

\section{FRI0714 LUPUS NEPHRITIS AND PROGNOSIS. EFFECT OF MEMBRANOUS AND OTHER COMPONENTS OF THE HISTOLOGY}

M.U. Martinez Martinez ${ }^{1}$, C. Vallín-Orozco ${ }^{1}$, H.E. Esparza-Holguin ${ }^{1}$, G. Aguilera-Barragán Pickens ${ }^{1}$, D. Martínez-Galla ${ }^{2}$, C. Abud-Mendoza ${ }^{1}$.

${ }^{1}$ Unidad de Investigaciones Reumatológicas; ${ }^{2}$ Renal Pathology, Hospital Central "Dr. Ignacio Morones Prieto", Universidad Autónoma de San Luis Potosí, San Luis Potosi, Mexico

Background: In 1983 Austin et al. informed a series of prognostic factors (including histology) associated with the development of renal failure in patients with lupus nephritis (LN). Differences in actual therapies may have different hazard ratios of renal failure than the described by Austin et al. ${ }^{1}$

Objectives: To evaluate histological factors associated with a decline in kidney function (DKF) in patients with SLE.

Methods: We evaluated all the patients in whom a kidney biopsy was performed. DKF was defined as a glomerular filtration rate (GFR) of less than $60 \mathrm{ml} / \mathrm{min} / \mathrm{m}^{2}$ in two determinations in the follow-up. Histology was graded according to Austin et al. ${ }^{1}$ (activity and chronicity) by a renal pathology specialist. Factors associated with the development of DKF were evaluated through Kaplan-Meier curves and Cox regression analysis (bivariate and multivariate).

Results: At this moment, we have followed 170 patients with LN and kidney biopsy, $130(76.5 \%)$ women, mean age at kidney biopsy was $29.7 \pm 13.2$ years classes of LN were: 71 patients $(41.8 \%)$ class IV, $30(17.6 \%)$ class V, $22(12.9 \%)$ class III/V, $19(11.2 \%)$ class IV/V, $16(9.4 \%)$ class III, and other classes 12 patients; 135 patients $(79.5 \%)$ have a minimum follow-up of 12 months. There were statistically significant differences in four groups of $L N$ : pure proliferative (classes III or IV), the combination with membranous (III/IV $\pm \mathrm{V}$ ), pure membranous (V) or other classes (Figure1).

Table 1. Factors associated with a DKF

\begin{tabular}{lcccc}
\hline Histological feature & $\begin{array}{c}\text { Bivariate } \\
\mathrm{HR}(\mathrm{Cl})\end{array}$ & $\begin{array}{c}\text { Bivariate } \\
\mathrm{p} \text {-value }\end{array}$ & $\begin{array}{c}\text { Multivariate } \\
\mathrm{HR}(\mathrm{Cl})\end{array}$ & $\begin{array}{c}\text { Multivariate } \\
\mathrm{p} \text {-value }\end{array}$ \\
\hline $\begin{array}{l}\text { Glomerular abnormalities } \\
\quad \text { Cellular proliferation }\end{array}$ & $1.01(0.83-1.22)$ & 0.920 & $\mathrm{NA}$ & $\mathrm{NA}$ \\
$\quad$ Karyorrhexis & $0.94(0.76-1.17)$ & 0.566 & $\mathrm{NA}$ & $\mathrm{NA}$ \\
$\quad$ Celular crescents & $1.09(0.96-1.22)$ & 0.179 & $0.95(0.82-1.10)$ & 0.501484 \\
$\quad$ Hyaline thrombi & $0.85(0.64-1.12)$ & 0.248 & $0.65(0.47-0.90)$ & 0.008837 \\
$\quad$ Leukocyte infiltration & $1.23(0.97-1.57)$ & 0.0848 & $1.03(0.77-1.40)$ & 0.815447 \\
$\quad$ Glomerular sclerosis & $1.79(1.41-2.26)$ & $<0.001$ & $1.67(1.23-2.25)$ & 0.000838 \\
$\quad$ Fibrous crescents & $1.71(1.27-2.29)$ & $<0.001$ & $1.55(1.11-2.16)$ & 0.009917 \\
$\quad$ Membranous & $0.50(0.30-0.85)$ & 0.0105 & $0.48(0.27-0.88)$ & 0.017001 \\
Tubulointerstitial abnormalities & & & & \\
$\quad$ Interstitial cell infiltration & $1.31(1.13-1.56)$ & $<0.001$ & $1.18(0.95-1.47)$ & 0.126099 \\
$\quad$ Interstitial fibrosis & $0.80(0.92-1.70)$ & 0.162 & $0.74(0.47-1.16)$ & 0.194012 \\
$\quad$ Tubular atrophy & $1.33(1.02-1.74)$ & 0.035 & $1.10(0.47-1.17)$ & 0.613154 \\
\hline
\end{tabular}

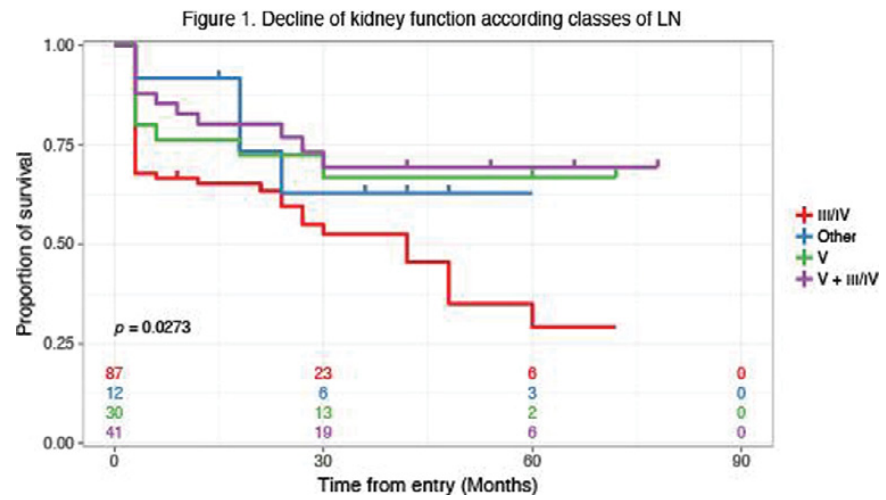

